\title{
The effect of body mass index on postoperative pain in paediatric surgical population
}

\author{
Ntritsou V., Papagiannopoulou P., Stefanović D., Karapetyan A., \\ Mademli A., Zachariadou C.

\section{Anesthesia Department, «G. Gennimatas» General Hospital, Thessaloniki, Greece}

\section{Introduction}

In children body mass index (BMI) varies with age and sex. WHO defines childhood overweight and obesity according to standard deviations (BMI Z-scores) from the mean BMI. The aim of this study is to evaluate the effect of BMI on postoperative pain and postoperative analgesia requirements in paediatric surgical population.

\section{Materials and methods}

757 consecutive children, aged 1 to 15 years old, ASA PS I-III, were included in this study.

Participants were allocated into four groups according to WHO BMI classification:

Group $1(n=110)$ underweight,

Group $2(n=456)$ normal weight,

Group $3(n=106)$ overweight and

Group $4(n=85)$ obese.

All patients underwent elective surgical procedures under general anesthesia and received 0.003$0.005 \mathrm{mg} / \mathrm{kg}$ fentanyl, $20 \mathrm{mg} / \mathrm{kg}$ paracetamol and $0.02 \mathrm{mg} / \mathrm{kg}$ nalbuphine intraoperatively according to standard department protocol. Demographic data were obtained from patients records.

Postoperative pain scores with Hanallah, NRS and Wong Baker (WBFPS) pain scales at rest and movement (deep breath) and Aldrete score were assessed in 1(st) hour following surgery. Postoperative analgesic requirements after discharge were recorded.

Statistical analysis was performed with ANOVA posthoc and chi square tests. Level significance was set at $p<0.05$.

Table 1. Patient's demographic data

\begin{tabular}{llcccc} 
& & Group 1 & Group 2 & Group 3 & Group 4 \\
\hline Age & Mean & 6.3 & 8.7 & 9.5 & 8.2 \\
& SD & 3.3 & 3.6 & 2.9 & 3.0 \\
BMI & Mean & $\mathbf{1 2 . 9}$ & $\mathbf{1 6 . 6}$ & $\mathbf{2 0 . 5}$ & $\mathbf{2 3 . 2}$ \\
$\mathbf{p}<\mathbf{0 . 0 5}$ & SD & $\mathbf{1 . 5}$ & $\mathbf{2 . 6}$ & $\mathbf{2 . 9}$ & $\mathbf{4 . 5}$ \\
ASA PS (I/II/III) & $82 / 28 / 0$ & $363 / 81 /$ & $70 / 34 / 2$ & $61 / 19 / 5$ \\
\hline $\begin{array}{l}\text { Duration } \\
\text { anaesth }\end{array}$ & Mean & 68.2 & 70.4 & 75.4 & 75.2 \\
Duration & Mean & 49.4 & 51.6 & 60.3 & 55.0 \\
surgery & SD & 28.5 & 30.2 & 27.2 & 24.4 \\
FNT & Mean & 4.8 & 5.2 & 4.1 & 3.9 \\
( $\boldsymbol{\mu g} / \mathbf{k g})$ & SD & 3.2 & 2.5 & 1.8 & 2.3 \\
\hline
\end{tabular}

\section{Conclusion}

BMI may relate with immediate postoperative pain scores and analgesia. Underweight and normal children received less analgesics and reported less pain compared to overweight and obese children, postoperatively.

Group 1 Group 2 Group 3 Group 4 p- value

Mean Mean Mean Mean

$\pm S D \quad \pm S D \quad \pm S D \quad \pm S D$

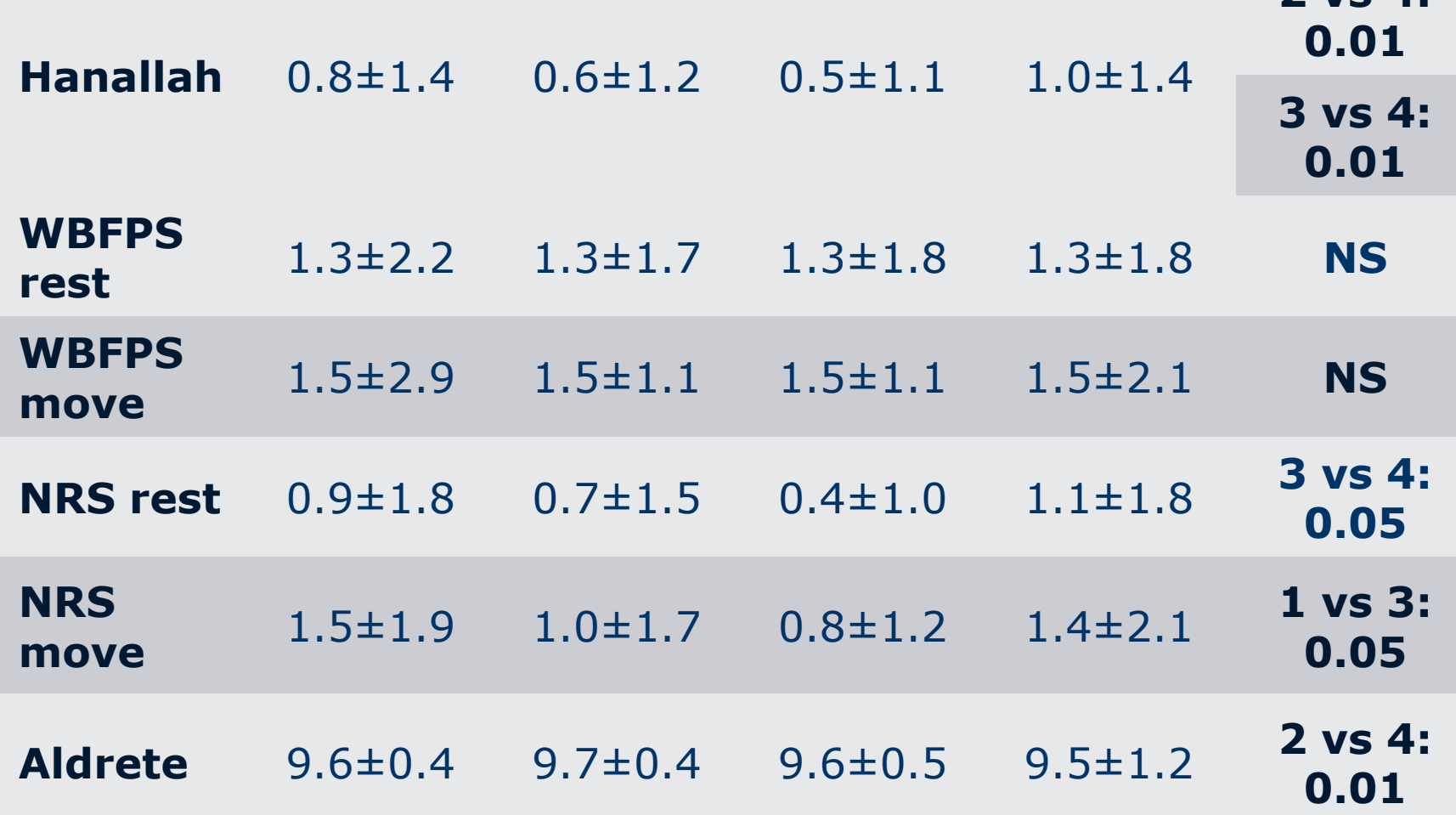

vs 4:

vs 4

vs 4:

vs 3

0.01 